\title{
Immediate Intervention Effects of Standardized Multicomponent Group Interventions on People with Cognitive Impairment: A Systematic Review
}

\author{
Dominik Özbe*, Elmar Graessel, Carolin Donath and Anna Pendergrass \\ Center for Health Service Research in Medicine, Department of Psychiatry and Psychotherapy, \\ University Clinic Erlangen, Friedrich-Alexander-University Erlangen-Nürnberg, Erlangen, Germany
}

Handling Associate Editor: Isabelle Rouch

Accepted 19 November 2018

\begin{abstract}
.
Background: There is no curative medical treatment for dementia. Therefore, researchers turned their attention to nonpharmacological treatment approaches. Many reviews analyzed the efficacy of single-component interventions, but there has been no systematic review of multicomponent interventions so far.

Objective: The aim was to systematically review studies using standardized multicomponent group interventions for persons with dementia or persons with mild cognitive impairment (MCI) and to analyze their immediate intervention effects.

Methods: The databases PubMed, PsycINFO, PSYNDEX, and CINAHL were systematically searched. We included randomized controlled trials with people with MCI or dementia, which implemented interventions with at least two components that targeted different outcome domains. Additionally, the intervention had to be standardized and in a group setting.

Results: Nine studies met the inclusion criteria with a total sample size of $\mathrm{N}=513$ participants. Six studies applied two, two studies applied three, and one study applied four components. Four studies, which combined at least a physical and a cognitive component, had a positive effect on non-cognitive symptoms of dementia. Two of these interventions additionally had a positive effect on cognitive abilities. One study reported a positive effect on activities of daily living and another study showed an effect on quality of life.

Conclusion: In spite of the heterogeneity of the studies, multicomponent interventions suggest a positive effect on noncognitive symptoms, especially the combination of cognitive and physical components. Single studies had also an effect on additional outcome domains. By trend the effects are dependent on application rate and used assessments.
\end{abstract}

Keywords: Behavioral symptoms, cognitive dysfunction, dementia, multicomponent intervention, review

\footnotetext{
*Correspondence to: Dominik Özbe, MS, Center for Health Service Research in Medicine, Department of Psychiatry and Psychotherapy, University Hospital Erlangen, FriedrichAlexander-University Erlangen-Nuremberg, Schwabachanlage 6, 91054 Erlangen, Germany. Tel.: +49 913185 34642; E-mail: dominik.oezbe@uk-erlangen.de.
}

\section{INTRODUCTION}

Dementia often begins with a pre-clinical stage of mild cognitive impairment (MCI) and is followed by declines in multiple clinical domains including cognitive functioning, activities of daily living (ADL), and mood [1]. Because curative medical treatments are still not available [2], researchers have 
turned to wide arrays of non-pharmacological therapies to treat persons with dementia (PwD), including cognitive stimulation [3]; physical activity interventions [4] or other measures like music therapy [5]; sensory stimulation [6,7]; and dance movement therapy [8]. Two types of non-pharmacological therapies have been identified in the literature: single component treatments which rely on single targeted therapeutic approach such as cognitive stimulation or physical activity, and multicomponent approaches which combine two or more treatment strategies. Olazarán et al. [9] assessed the efficacy of 179 studies on non-pharmacological therapies and found that single component interventions affected the outcome domains they targeted, but showed nearly no effects on other domains. Although Olazaran et al. [9] did not systematically search for and review multicomponent interventions, they suggest that multicomponent interventions may have greater efficacy in that they generate broader positive outcomes. This is analogous to the family caregiving literature where multicomponent interventions have been the norm because of the multidimensional nature of the challenges faced by caregivers [10].

The goal of this paper is to conduct a systematic review of multicomponent interventions for persons with cognitive impairment. The specific aims are to: 1) systematically review studies with PwD or Persons with MCI (PwMCI) reporting standardized multicomponent interventions as group therapies; 2 ) illustrate these studies and analyze their risk of bias; 3) investigate their different combinations of components; and 4) analyze their efficacy on different outcome domains from baseline testing to the first measure point immediately after the intervention was finished.

\section{METHODS}

\section{Eligibility criteria (PICO)}

Relevant records for this review were randomized controlled trials of non-pharmacological multicomponent interventions for persons with any type of dementia or PwMCI. We defined multicomponent interventions as interventions with at least two components. In addition, these components should target different domains so that they are able to affect different outcome domains. Interventions had to be directed at either the PwD/PwMCI or at the $\mathrm{PwD} / \mathrm{PwMCI}$ and their caregivers simultaneously, and had to be held in a group setting. We chose to exclude individual interventions, because they are not comparable to group interventions. PwD/PwMCI could be home-dwelling or institutionalized. Waitlist control groups, placebo or active control groups receiving only single component interventions, and no treatment or usual care were used as control conditions. Studies using anti-dementive drugs as basic medication for both groups were allowed. For a study to be selected, it had to report outcomes for the PwD/PwMCI. We included every study that was published until March 27th, 2017. Only publications in peer-reviewed journals in English and German language were considered.

Studies were excluded if the sample was mixed with the healthy elderly, because the mix-up can distort the intervention effects in comparison to a group where every member has a degree of cognitive impairment. To be considered the goal of a study had to be the treatment of cognitive impairment. If it was the sole objective to treat another psychological disorder such as depression, the study was excluded. Interventions that vary significantly from group to group, because they are individualized, tailored or have an innate focus on individual needs, like psychotherapy or occupational therapy were excluded. Therefore, we only included standardized interventions that were held in a group. Standardization is necessary to describe the intervention in detail and therefore to avoid the "black box phenomenon" [11]. This is necessary to guarantee an experimental study design with the criteria of repeatability. Individualized support of the participants would furtherly increase this problem.

\section{Search strategy}

A systematic literature search was conducted using the electronic databases PubMed, PsycINFO, PSYNDEX, and CINAHL. There is no universally used term for multicomponent interventions. Hence, reviews and articles regarding our research questions were examined to identify widely used terms for our literature search. In a next step a project workgroup gathered possible key words and synonyms. Afterwards, the complete set of search terms was evaluated and completed by four international experts in this field of research (E.G., H.B., M.M., and R.S.). Due to the multidimensional nature of the interventions and therefore, their expected effects on multiple outcomes, we did not define a specific outcome.

The search terms were adjusted for each database according to the individual thesauruses and 
Table 1

PubMed search terms

"dementia" [MeSH Terms] OR dement* OR alz* OR alzheimer OR "lewy body" OR "memory impairment" OR "memory disorder" OR "cognitive impairment" OR "cognitive disorder" OR "memory dysfunction" OR

"cognitive dysfunction" OR "mild cognitive impairment" OR "MCI"

AND Combine OR combined OR combining OR combination OR multidisciplinary OR multi\#disciplinary OR multicomponent OR multi\#component OR multimodal OR multi\#modal OR multifaceted OR multi\#faceted OR multidimensional OR multi\#dimensional OR multiple OR adjunction OR adjunctive OR interdisciplinary OR cross-disciplinary OR multifactorial OR multi\#factorial

AND therapy OR therapies OR intervention OR interventions OR treatment OR treatments OR training OR "cognitive training" OR "cognitive support"

AND "Randomized Controlled Trial" [Publication Type] OR "Randomized Controlled Trials as Topic"[Mesh] OR "rct" OR controlled trial OR control trial OR randomized controlled trial OR randomised controlled trial OR randomized control trial OR randomised control trial

Notes. Not specified terms were searched in title or abstract.

limitations. The exact search terms used in PubMed are shown in Table 1.

Study selection, data extraction, and analysis

After importing the records of the four electronic databases to the licensed EndNote library, duplicates were deleted. Three independent raters (D.Ö., C.V., and M.B.) screened titles and abstracts depending on the inclusion and exclusion criteria. Cases of disagreement were solved in discussion. The remaining articles were completely read by one author (D.Ö.) and rated for suitability. Cases of ambiguity were discussed with E.G. and A.P.

Before extracting data, included studies were assessed for risk of bias applying the Cochrane Collaboration's tool for assessing risk of bias [12], judging a high, low, or unclear risk of bias over six domains. Of the information given in the included articles, the following data items have been extracted: sample size $(n)$; mean age; baseline Mini-Mental State Examination (MMSE) scores; setting; diagnosis; medication; adverse events; length of intervention (months); exercise protocol (length and frequency of exercise sessions); group size; content and intensity of the single components and effects on all outcomes that have been measured at baseline and at the first measure point after the intervention was concluded.

Afterwards, we conducted a qualitative analysis of the included studies. In our analysis, we only consid- ered outcomes that were assessed at baseline and at the first time point after completion of the intervention for the whole group of participants. We refrained from a quantitative analysis because of the extreme heterogeneity in used components and content, and in both the intervention length and in exercise protocol.

\section{RESULTS}

We found 2,764 articles through the systematic search (see Fig. 1). After the removal of duplicates, 2,573 titles and abstracts were screened. From this sample, 167 full-texts were assessed for eligibility. Three studies were excluded because inclusion criteria could not be applied and the authors of the article were not available for clarification [13-15]. In the final analysis, nine articles were included.

The included studies (Table 2) took place worldwide: Germany $(n=3)$; USA $(n=1)$; Italy $(n=2)$; Spain $(n=1)$; Canada $(n=1)$; and Japan $(n=1)$. The studies comprised a total of $\mathrm{N}=513$ participants, of which $312(61 \%)$ were women and 201 (39\%) were men. Seven studies were conducted with community-dwelling individuals, one was conducted with community-dwelling care dyads and one took place in a nursing home. The included studies ranged in mean age between $70.3( \pm 5.8)$ years and 85.1 $( \pm 5.1)$ years. Three studies conducted their intervention only with persons with MCI. Four studies included only participants with a possible or probable diagnosis of $\mathrm{AD}$ or a primary degenerative dementia and two studies accepted persons with either MCI or probable AD as participants. Participants' levels of cognitive impairment, as measured by the MMSE at baseline, ranged from a mean of $13.8( \pm 5.4)$ points, which indicates moderate cognitive impairment, to $28.5( \pm 1.0)$ points, indicating mild cognitive impairment.

\section{Component classification}

In our systematic review, we found six different domains of which components can be assigned: cognitive interventions; physical interventions; ADL training; relaxation skill; dual task training; and a recreational intervention. We assigned interventions to the different domains as follows.

Cognitive interventions ranged from cognitive training, to cognitive stimulation, to reminiscence therapy, to psychoeducation. We defined cognitive training according to Gates and Valenzuela [16] as interventions with repeated practice on inherently 


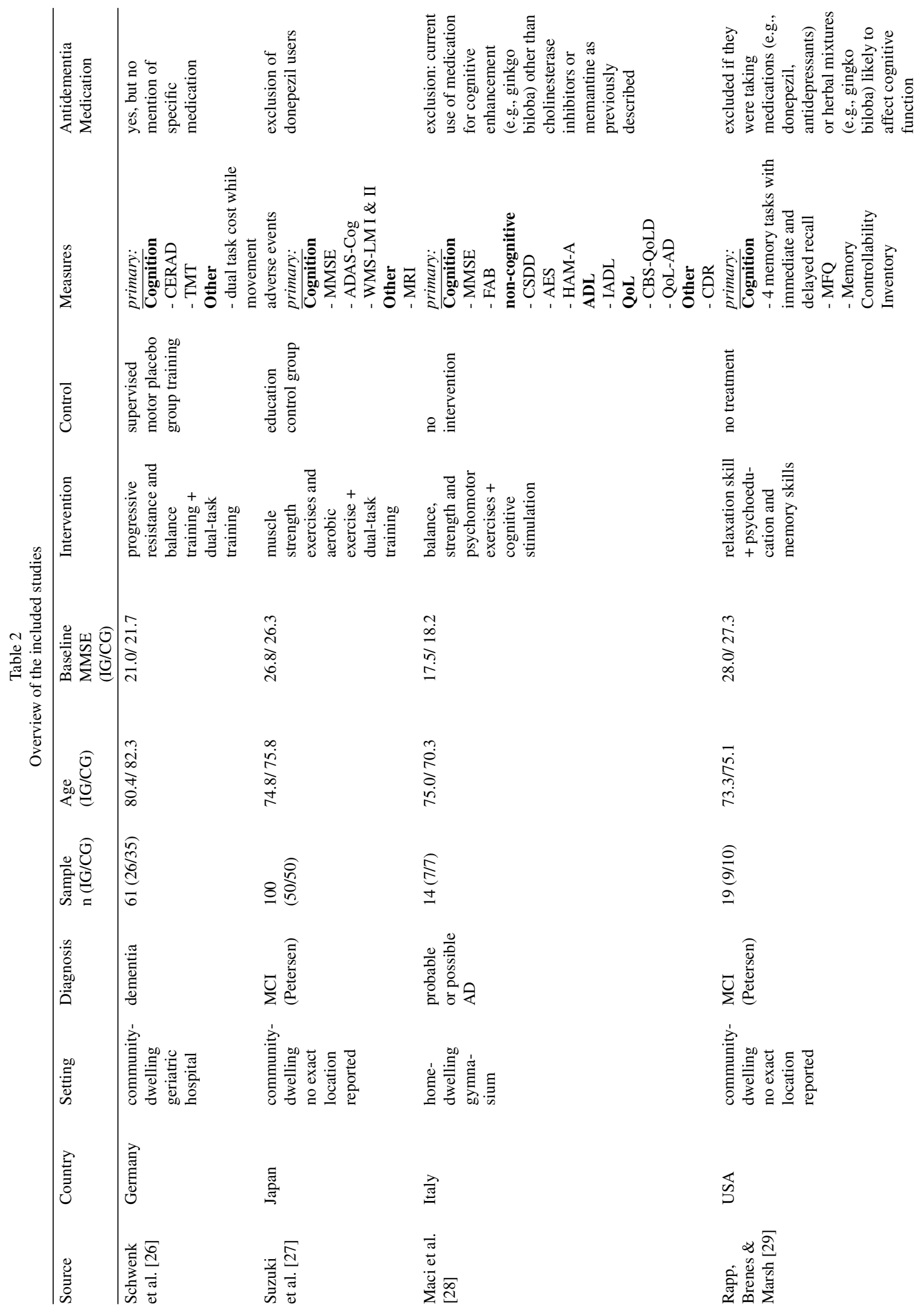




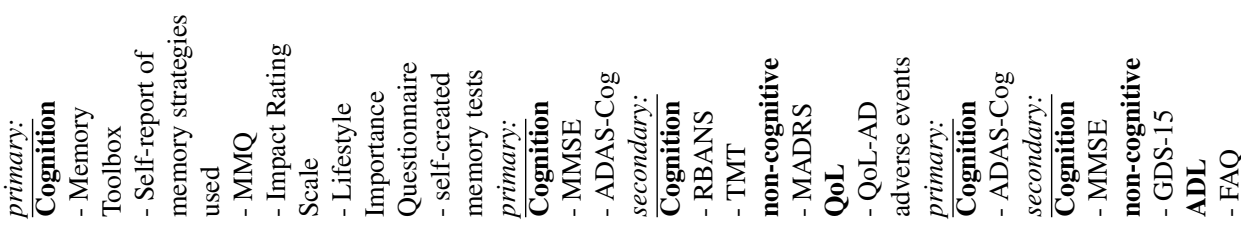
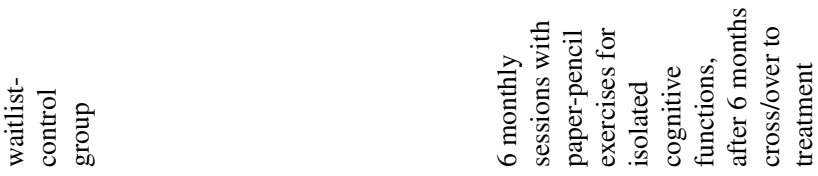

๕.
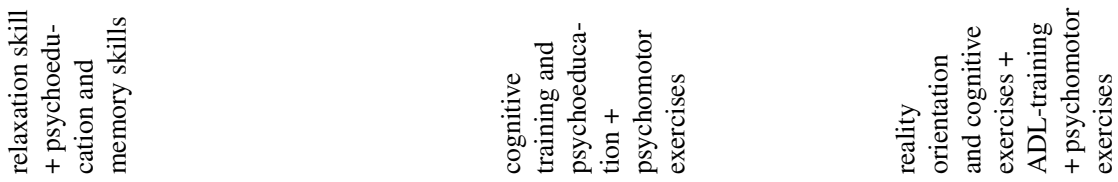

$n$
$\infty$
ñ
ñ

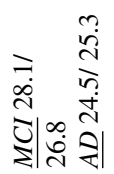

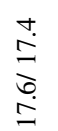

$\underset{\substack{i \\ i}}{\stackrel{\infty}{0}}$

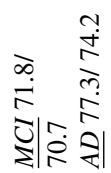

ले

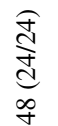

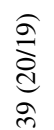

$\underset{\substack{+ \\ \text { 辛 }}}{\stackrel{0}{d}}$

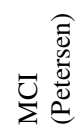

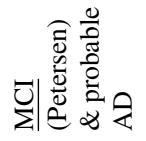

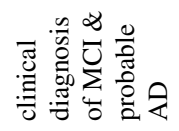

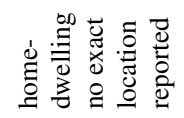

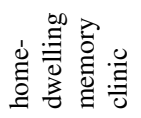

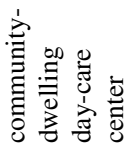

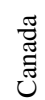

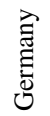

कूँ

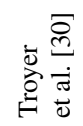

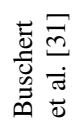

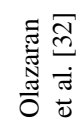




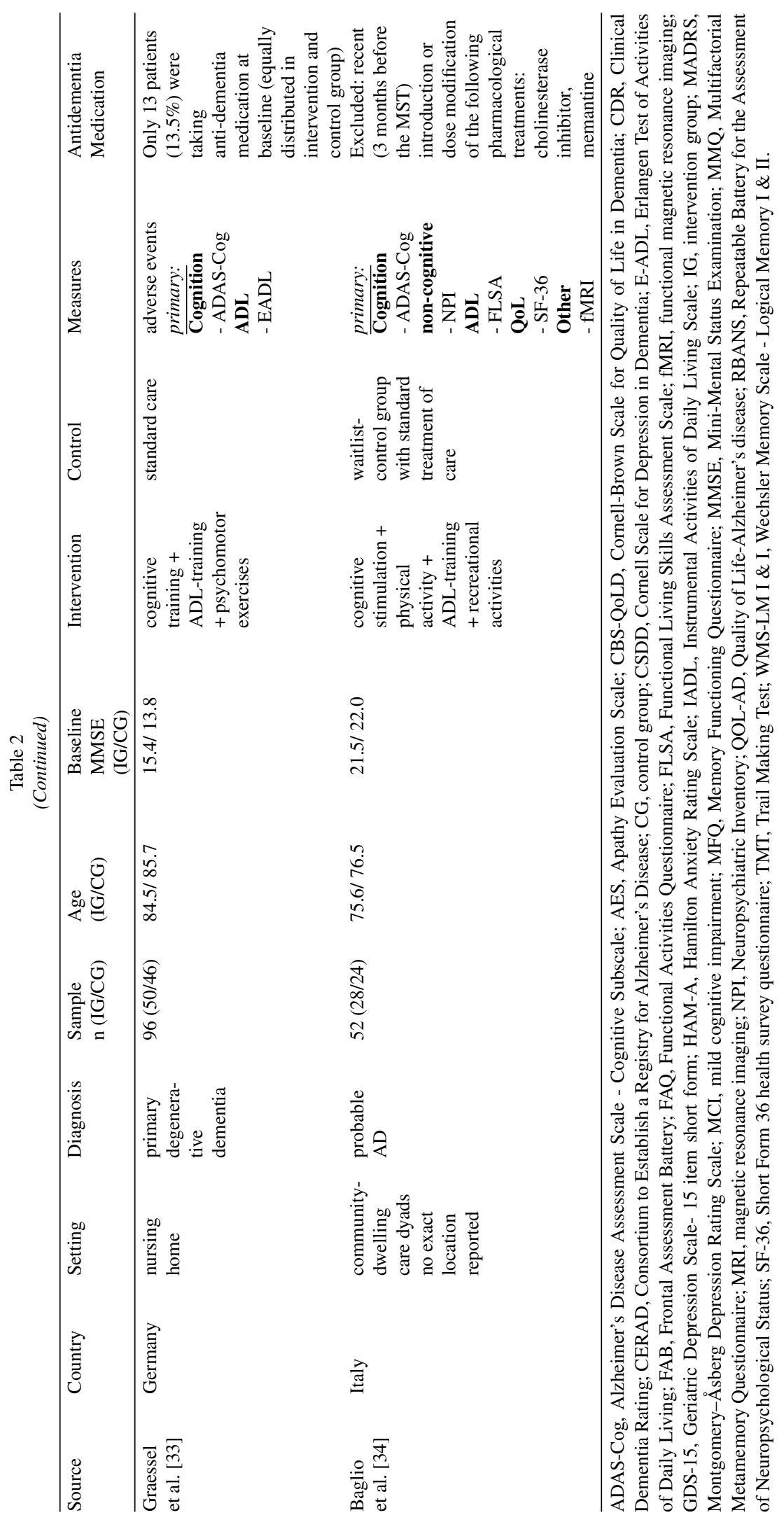




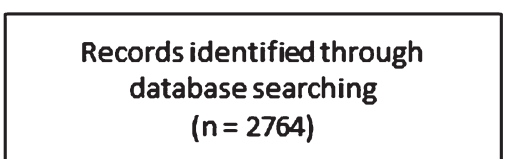

( $n=2764)$
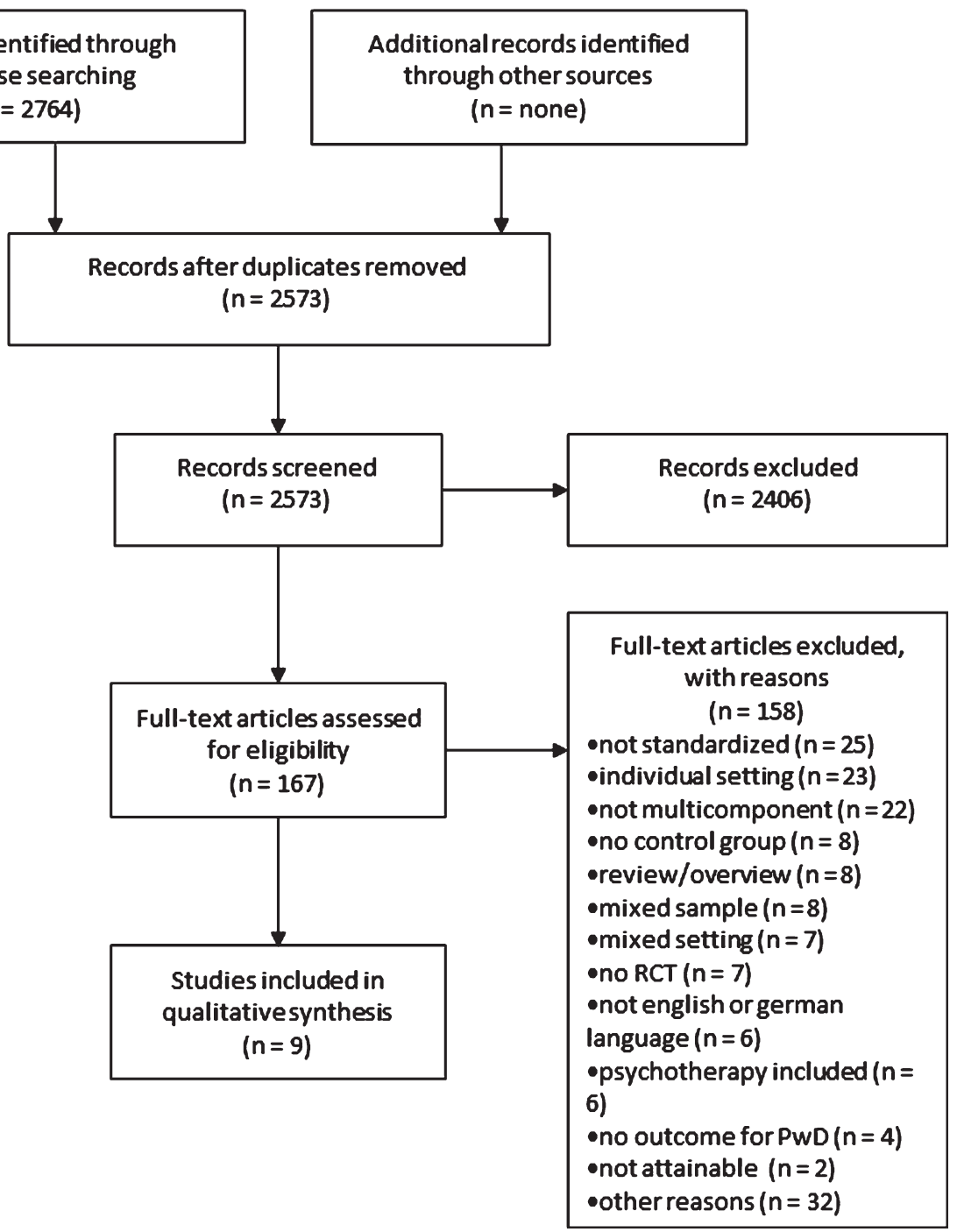

Fig. 1. PRISMA flow chart.

problematic tasks, applying standardized tasks that target certain cognitive domains. With this definition, we summarized interventions like memory strategy training and repetitive cognitive exercises. We used Clare and Woods (2008; cited in [17]) definition for cognitive stimulation. Cognitive stimulation aims at cognitive and/or social function. It uses cognitive activities which do not focus on practicing specific cognitive domains and includes a social element. Additionally, reality orientation sessions are subsumed under the definition of Clare and Woods (2008; cited in [17]). Reminiscence therapy was defined as discussions and activities that involve past activities, events and experiences, and may be aided by the use of prompts [18]. Furthermore we subsumed psychoeducation under cognitive interventions. It is a specific form of stimulation, which aims at minimizing patients' knowledge gaps concerning their illness, through the imparting of knowledge [19].

The National Institute on Aging distinguishes physical interventions into endurance exercises, strength exercises, and balance or flexibility exercises [20]. Additionally, we subsumed psychomotor interventions under this category. Psychomotor interventions simultaneously utilize cognitive and motor stimulation [21].

ADL training aims to improve, or stabilize, a subject's ability to execute ADL. Typical exercises are 
dressing oneself, washing hands, cooking, or using medication [22].

With relaxation skills, individuals learn procedures that induce a state of reduced musculoskeletal activity [23].

Dual-task trainings are interventions with the aim of maintaining normal gait while the individual performs other cognitive tasks [24]. These tasks could be calculating or reciting poems, which are not associated with the movement.

A recreational intervention uses recreation and activity based interventions in a systematic process to promote psychological and physical health, recovery, and well-being [25]. This could be activities like painting, pottery, or playing a card game together.

Most commonly, studies used two or three different components. Only one study implemented four components. Cognitive and physical components were most frequently used (seven of nine studies). Therefore, combinations of these two components were the most prevalent. The group size ranged from four to six participants in the smallest group intervention, to 16 to 17 participants in the biggest group intervention. Every intervention used elements which were meant to integrate their participants into the groups and strengthen cohesion. This could be elements such as group discussion, singing songs together, or the encouragement to talk to each other. Figure 2 shows an overview of the components that were used in a study. The included studies combined the components in the following fashion:

\section{Two-component interventions}

Participants in Schwenk et al.'s [26] intervention first underwent progressive resistance and balance training until they could walk 10 meters and perform basic balance exercises without support. This lasted for 60 min every session and was interrupted by breaks. Within each group session subjects engaged in 15 min group dual-task training and additional individual dual-task training for 10-15 min.

One session of Suzuki et al.'s [27] intervention comprised $10 \mathrm{~min}$ warm-up and stretching, $20 \mathrm{~min}$ muscle strength exercises, and $60 \mathrm{~min}$ of aerobic exercise, postural balance training and dual-task training. Special features were eleven sessions with 20-30 min outdoor walking and prescribed home-based exercises with self-monitoring through a booklet and a pedometer.

The $240 \mathrm{~min}$ sessions of Maci et al. [28] were divided in $60 \mathrm{~min}$ transportation to and from the inter- vention, $60 \mathrm{~min}$ of physical activation consisting of balance, strength, and psychomotor exercises, $60 \mathrm{~min}$ of cognitive stimulation, $30 \mathrm{~min}$ of group discussion, and $30 \mathrm{~min}$ of break.

The workgroup of Rapp et al. [29] implemented their intervention as follows: The first session was used for learning a relaxation skill (systematic breathing) and psychoeducation. In the remaining sessions, different memory skills were taught and practiced. In addition, homework regarding the trained skill was assigned.

Troyer et al.'s [30] intervention had the following structure: The first $60 \mathrm{~min}$ of a session were used for psychoeducation, or teaching a relaxation skill (deep-breathing technique). In the second $60 \mathrm{~min}$, participants learned and practiced memory skills.

A typical session of Buschert et al. [31] comprised 40 min of cognitive training, 10 min of psychomotor exercises, $10 \mathrm{~min}$ of psychoeducation, $10 \mathrm{~min}$ of break, and $20 \mathrm{~min}$ for arriving and warm-up, as well as for parting.

\section{Three-component interventions}

Olazaran et al.'s [32] sessions devoted $20 \mathrm{~min}$ to welcome and conclusion, $50 \mathrm{~min}$ to reality orientation, $30 \mathrm{~min}$ to cognitive exercises, $30 \mathrm{~min}$ to ADL-training, $50 \mathrm{~min}$ to psychomotor exercises, and 30 min to coffee breaks.

A typical session of Graessel et al.'s [33] intervention comprised $10 \mathrm{~min}$ of introduction, $30 \mathrm{~min}$ of psychomotor exercises, $10 \mathrm{~min}$ of break, $30 \mathrm{~min}$ of cognitive training, and 40 min of ADL-training.

\section{Four-component intervention}

A session of Baglio et al.'s [34] intervention was structured as follows: $45 \mathrm{~min}$ of cognitive stimulation, $30 \mathrm{~min}$ of physical activity, $30 \mathrm{~min}$ of ADL-training, and 45 min of recreational activities.

\section{Intervention effects}

The included studies reported the following five different outcome domains on which they assessed effects of their intervention: cognition; non-cognitive symptoms; ADL; quality of life (QoL); and "other".

Every study used at least one measure to assess cognition. The most widely used measures were the MMSE (4 studies) and the Alzheimer's Disease Assessment Scale - Cognitive Subscale (ADAS-Cog) (5 studies). They assess different cognitive abili- 


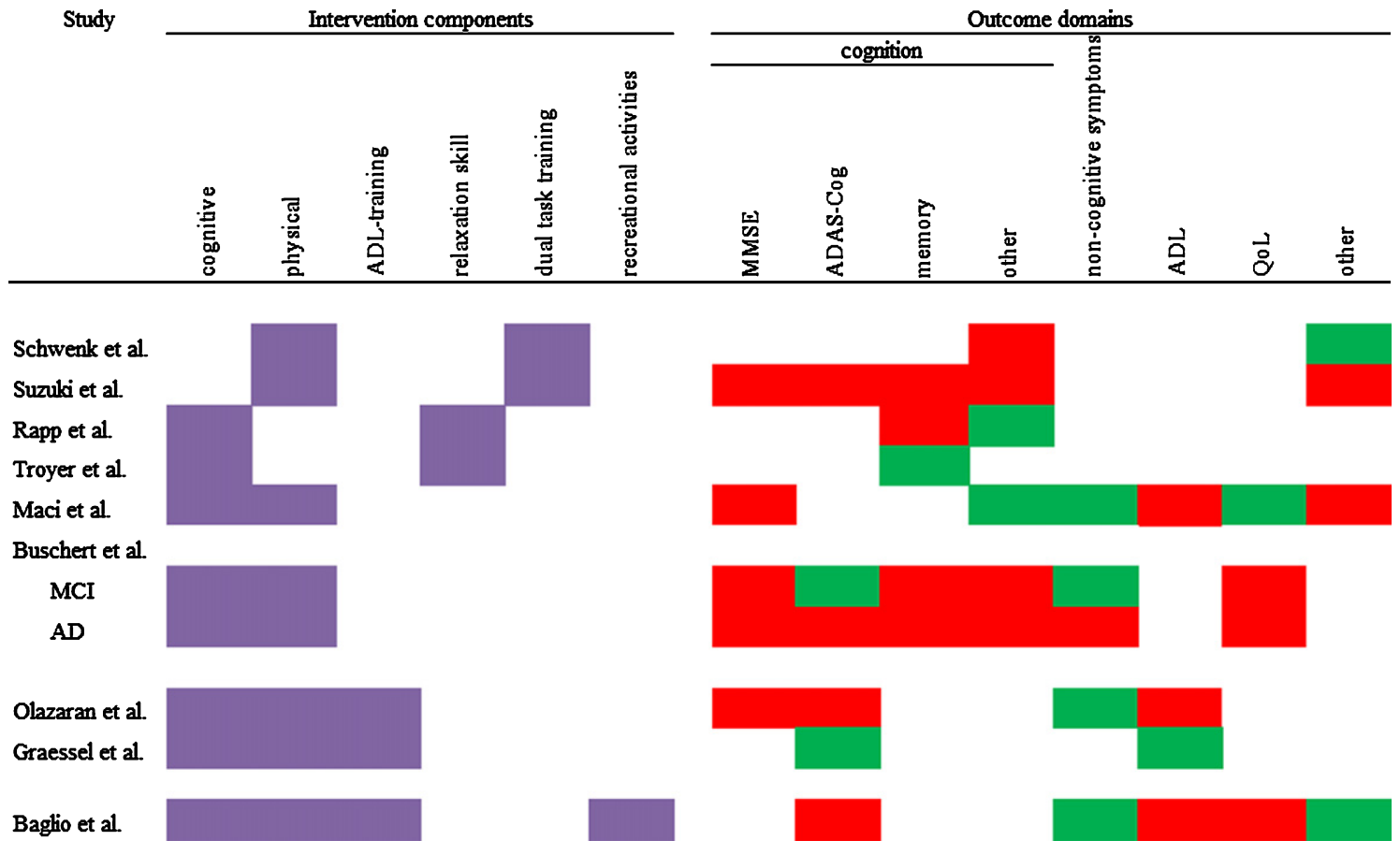

Fig. 2. Overview of used components and intervention effects on different outcome domains. Purple = component used; red = IG has the same or worse values than CG; green = at least one measure in this domain shows an advantage for the IG.

ties and come up with a global cognition score. As these measures are commonly used in the literature, we examined them separately. Four studies assessed memory capacities with four different test procedures. Another four studies used different tests or let the participants assess their cognitive abilities themselves.

Four studies measured non-cognitive symptoms. This domain subsumes scales that examine psychological symptoms. These symptoms could be for example: depression; anxiety; apathy; or agitation.

ADL were assessed by four studies. Three studies used assessment by proxy to examine ADL, while one study used a performance test to measure the ability to execute ADL.

The "other" domain subsumes tests not typically used in intervention studies with PwD and PwMCI. On the one hand, these are imaging techniques that were used in two studies. On the other hand, there was one study that developed a specific test for their intervention. Additionally there was one study that used a rating scale to assess dementia on multiple outcome domains.

Subsequently, we will present the immediate intervention effects of the included studies.

\section{Two-component interventions}

Schwenk et al. [26] found no effects in the CERAD (cognition domain) and no effect on dual task cost of a serial 2 forward calculation while walking ( 7 measures, "other" domain). They reported 5 significant effects in 7 measures $(p<0.001 ; p=0.007 ; p=0.001$; $p=0.056 ; p=0.003 ; p=0.222 ; p=0.026)$ of dual task cost of serial 3 backward calculation while walking ("other") using rm-ANOVAs.

Suzuki et al. [27] found no significant effects of their intervention, when they assessed the whole participant group with MCI. They used rm-ANOVAs for their statistical operations.

Maci et al. [28] reported that there were no significant effects on MMSE (cognition), Instrumental Activities of Daily Living Scale (ADL), and Clinical Dementia Rating ("other"). Concerning the Frontal Assessment Battery (FAB) (cognition), the authors reported a significant deterioration in the control group (CG) $(p<0.05)$, while the intervention group (IG) did not change. Regarding the Cornell Scale for Depression in Dementia, Hamilton Anxiety Rating Scale, Apathy Evaluation Scale (each non-cognitive symptoms), Cornell-Brown Scale for Quality of Life 
in Dementia (CBS-QoLD), and QoL-AD (each QoL) the authors reported a significant improvement in the IG (all ps <0.05) while the CG did not change. The authors used Wilcoxon tests for comparing post-tests values with baseline values.

Rapp et al. [29] wrote that there were no significant effects on the four objective memory tasks and also no significant effects on the five subscales of the Memory Functioning Questionnaire (cognition). They found significant effects on two of four scales of the Memory Controllability Questionnaire $(p s<0.001)$. They used MANOVAs for statistical analysis.

According to Troyer et al. [30], there were no significant effects on memory-related affect and thoughts measured by 4 scales, or on objective memory ability measured by three tests. Regarding memory strategy knowledge and behavior, the authors reported significant group by time interactions on the Memory Toolbox questionnaire $(p<0.001)$, the strategy subscale of the MMQ $(p=0.021)$, and the used strategies while performing the objective tasks ( $p=0.049$; cognition). These interactions indicated as per the authors, a greater improvement for the IG. To analyze their data, the authors used rm-MANOVAs.

Buschert et al. [31] found no significant effects for PwD. For PwMCI they reported some significant effects. For the primary outcome ADAS-Cog (cognition), the authors reported a significant interaction of treatment by progression $(p=0.02)$, indicating a deterioration in the CG and no change in the IG. In the secondary outcome Repeatable Battery for the Assessment of Neuropsychological Status (cognition), they found significant main effects for the two subtests (both $p<0.01$ ). Additionally, a significant interaction effect treatment by progression $(p<0.01)$ was found for the secondary outcome MADRS (noncognitive symptoms), indicating an improvement in the IG and no change in the CG. Regarding the secondary outcomes, Trail Making Test (cognition) and QoL-AD (QoL), they reported no significant effects. They investigated the effects of the intervention with ANCOVAs.

\section{Three-component interventions}

Olazaran et al. [32] reported one significant intervention effect after 12 months. This was a group difference $(p=0.05)$ in the Geriatric Depression Scale- 15 item short form (non-cognitive symptoms) acquired using $t$-test. As the authors wrote, the IG tended to improve, while the CG tended to deterio- rate. They found no intervention effects on the other outcomes.

Graessel et al. [33] reported a significant effect of the intervention on the ADAS-Cog $(p=0.018$; $\operatorname{cog}$ nition) in a multiple regression, which indicates a deterioration of the $\mathrm{CG}$, while there was no change in the IG. They found a similar effect for the E-ADL $(p=0.015 ; \mathrm{ADL})$, again indicating a deterioration of the CG while there was no change in the IG.

\section{Four-component interventions}

Baglio et al. [34] found significant effects of their intervention on NPI ( $p=0.019$; non-cognitive symptoms), where the IG showed an improvement and the $\mathrm{CG}$ did not change. They also found significant intervention effects in fMRI ("other"). The IG showed an increased activation of the bilateral superior temporal gyrus (right $>$ left) and the right lentiform nucleus and thalamus $(p<0.05$ ). Additionally, the IG showed an increased activation in the right insular cortex with respect to the CG $(p<0.001)$. They used ANCOVAs to analyze the effects.

\section{Total intervention time analysis}

To better compare protocol length of the interventions, we computed a total intervention time score in min (months (with 4 weeks) $x$ frequency per week $x$ duration of a session) for every study. Total intervention time ranged from $720 \mathrm{~min}$ to $34560 \mathrm{~min}$ with a median of $3600 \mathrm{~min}$. Figure 3 gives an overview of the intervention protocol, the calculated total intervention time, and the group size in conjunction with the intervention effects. We divided the included studies concerning their total intervention time into tertiles. The low tertile included three studies up to a total intervention time of $2400 \mathrm{~min}$. The moderate tertile included three studies with a total intervention time above $2400 \mathrm{~min}$ up to a total intervention time of $4500 \mathrm{~min}$. The high tertile included three studies with a total intervention time above $4500 \mathrm{~min}$.

Assessing the low total intervention time interventions, we found two studies that reported effects on self-reported outcomes [29, 30]. And one study found positive effects on the ADAS-Cog and non-cognitive symptoms for PwMCI [31]. Of the interventions with moderate total intervention time, only one found no effect [27]. One study [26] reported one effect on the "other" domain and one study [34] found effects on two of the assessed outcome domains, namely on non-cognitive symptoms and the "other" domain. The 


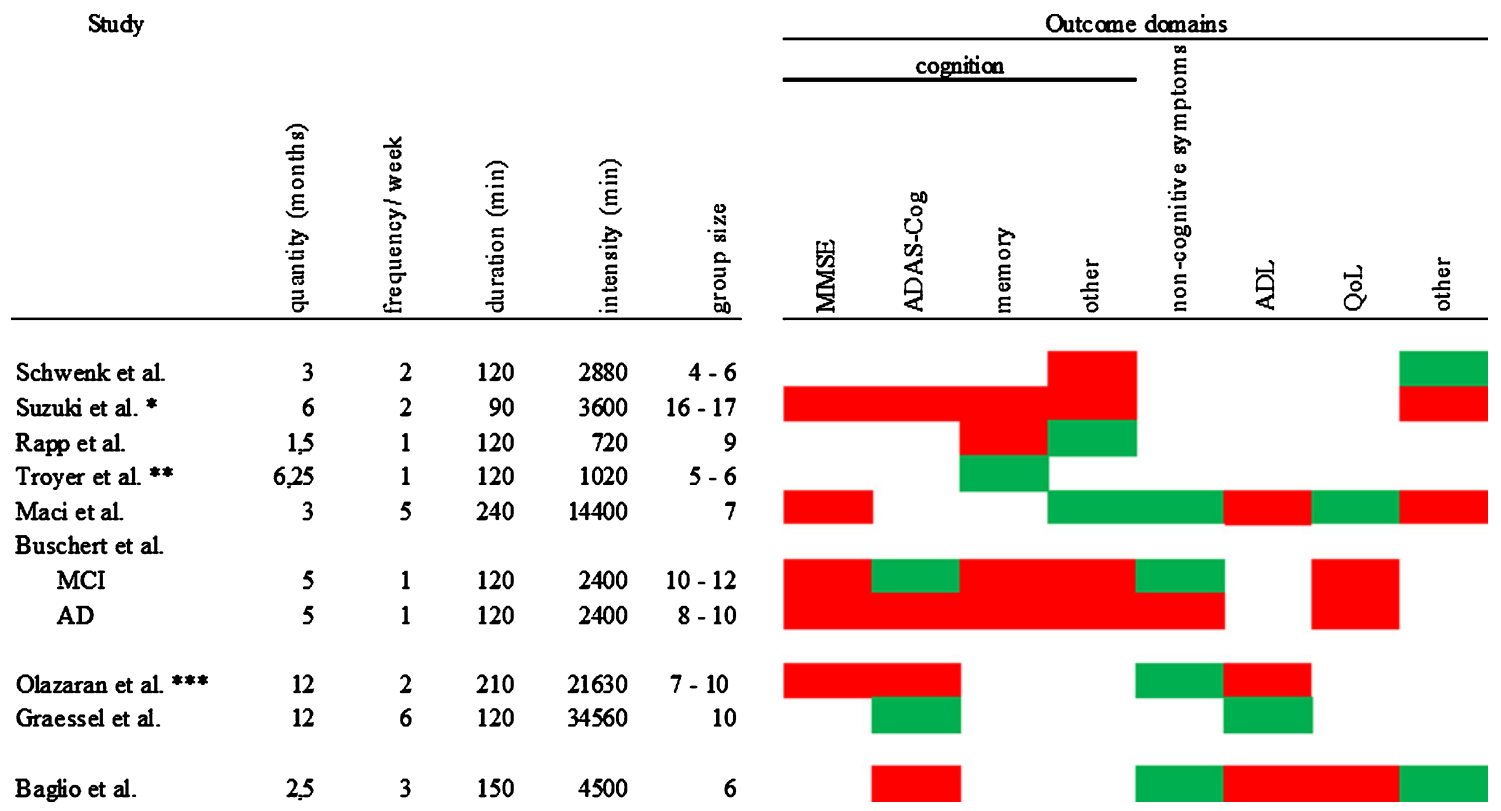

Fig. 3. Overview of intervention protocol, total intervention time and group size in conjunction with the intervention effects. Red $=$ IG has the same or worse values than CG; green = at least one measure in this domain shows an advantage for the IG; *the total intervention time was calculated with 40 reported sessions; ** the intervention had 10 sessions held as following: the first 7 sessions took place in 7 weeks followed by 3 review sessions: 1st after 2 weeks; 2 nd after additional 4 weeks, 3rd after additional 12 weeks; for the total intervention time calculation 180 min testing were subtracted; *** the total intervention time was calculated with 103 reported sessions.

affected outcomes were either assessed by objective others, or performance tests. All of the high total intervention time studies reported at least one positive effect on one outcome domain [32] (non-cognitive symptoms). One study [33] found effects on two outcome domains (cognition and ADL) and one even reported effects on three different domains (cognition, non-cognitive symptoms, and QoL) [28].

\section{Adverse events}

Three out of nine studies gave information about adverse events occurring in their trials [27, 32, 33]. Graessel et al. [33] reported 67 events and Suzuki et al. [27] reported 27 events. In both trials the authors found no significant group differences in the occurrence of adverse events. Olazaran et al. [32] reported only one event of psychological distress in the intervention group that was gone, without residual effects, after 3 months. They reported no adverse events in the control group.

\section{Risk of bias}

While assessing the risk of bias, we encountered several methodological flaws of the different studies. There was no study with an overall low risk of bias.
Only three studies did not get a high risk rating in any domain. The most problematic domains were selective reporting (four of nine studies had a high risk) and incomplete outcome data addressing (three of nine studies had a high risk; see Table 3). No study had a low risk rating in blinding of participants and personnel due to the character of non-pharmacological interventions where a blinding of an active participant and a delivering therapist is virtual impossible.

\section{Summary of effects}

Interventions combining physical components with dual task training (two-component) seem to have no global effect on cognition or other outcomes, but they can improve dual task performance. This effect only appeared in PwD, not in PwMCI. Cognitive interventions combined with a relaxation skill (two-component), appear to have only an effect on self-reported cognitive ability for PwMCI. For interventions which combined cognitive components with physical components and to some extent other components (two-, three-, or four-component), it looks as if there is a positive effect on non-cognitive symptoms in PwD and PwMCI. In PwD, this effect was observed in interventions taking place more than once a week (dosage dependency). Additionally, some of 
Table 3

Risk of bias assessment of the included studies

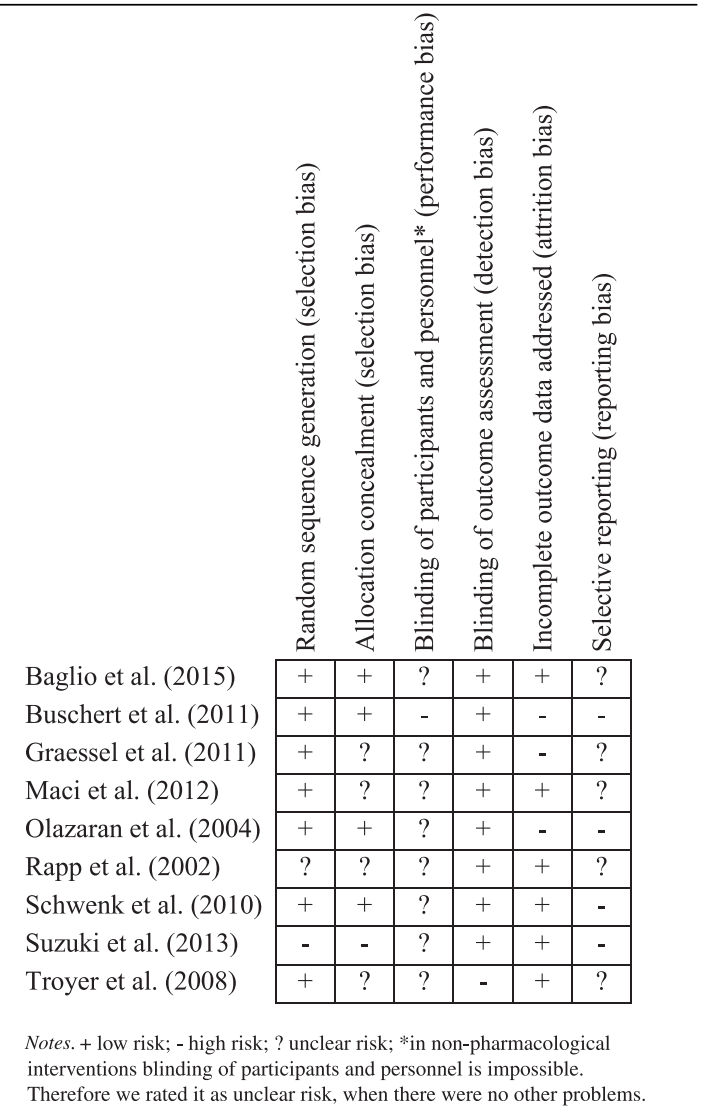

these interventions had a positive effect on cognition in performance tests. This effect could be achieved with an intervention frequency of once a week in PwMCI, while PwD seemed again to need a higher frequency. Only one out of three studies [28] found a positive effect on QoL, and one study out of four found a positive effect on ADL in a performance test [33].

\section{DISCUSSION}

The aims of this review were to systematically review and illustrate standardized multicomponent group interventions for PwMCI or PwD, and to evaluate their combinations of components and their efficacy on various outcomes. To the best of our knowledge, it is the first of its kind with this agenda. We refrained from quantitative analyses because of the vast heterogeneity of the interventions. Nine trials with two, three, or four components met our inclusion criteria. The trials preferred cognitive and physical components to be combined. One study took place in a nursing home, while eight studies had communitydwelling participants.

All studies assessed cognitive outcomes and three studies reported a positive effect on cognitive performance tests. Two studies reported a positive effect on self-reported cognition. Of the five studies that measured non-cognitive symptoms, four reported a benefit of the intervention in this domain. Concerning ADL, only one out of four studies found a positive effect. A similar pattern emerged for QoL. Only one out of three studies found a positive effect on QoL. One study found a reduction of dual task cost, a specifically created measure reflecting the used intervention, and one study reported an increase of activation in fMRI. Three studies looked at adverse events. Their findings suggest that multicomponent interventions are safe and without additional risks to the participants.

\section{Cognition}

Although cognition was assessed in every included study, positive effects on cognition were only found in three studies, [28, 31, 33]. One study [28] found an effect on the FAB but not on the MMSE. As it was a pilot study with a small sample size, many assessments and the sole study that used the FAB we refrain from further interpreting this finding. Additionally, two studies [31,33], which used the ADAS-Cog performance test showed positive findings. On the one hand, both studies took place in Germany and each had one cognitive and one physical component. But, they showed differences in following factors: number of components of the intervention and its frequency; setting; and participants. One possible interpretation why these different intervention studies were the only interventions that positively affected global cognition is that the scope of most of the other interventions was too narrow to globally affect cognition. This point could be represented by the finding that dual task training effectively reduced the workload of doing two tasks simultaneously [26], but it did not affect other cognitive outcomes in any way. Now one can question the value of an intervention which has specific and narrow effects, but no effect on global cognition. However, the capacity of doing two tasks simultaneously is argued to be protective against falls, [35] and therefore it is an important outcome for interventions with elderly people. Consequently, interventions that present only narrow effects may be of particular value. 
On the other hand, in this context, the chosen assessment seems to be relevant to show intervention effects. Positive effects were only shown in studies which used the ADAS-Cog. This could imply that the ADAS-Cog is more sensitive to change and that the MMSE might not be a suitable instrument to measure change in cognitive impairment, as it is only a screening test for the presence of cognitive impairment. The same opinion is held by Clark et al. [36] who found large measurement errors and considerable fluctuation in annual MMSE scores. They concluded that MMSE's value of measuring progression in Alzheimer's disease is limited if the observation period is less than three years.

Furthermore it has to be said, that the included studies primarily used global cognition scores, which has both advantages and disadvantages. An advantage is that these global cognition scores limit heterogeneity, which allows us to better compare the studies concerning their effects. As a drawback it is not possible for us to give differentiated information about the effects of multicomponent interventions on particular cognitive abilities or executive functions.

The positive results of two studies that reported an effect on self-reported cognition should be interpreted with caution. We think that a self-assessment of cognition by persons with cognitive impairment represents a source of bias, because cognitive impairment changes the capacity for self-assessment and thus constitutes an impairment of reliability of measurement.

Comparing our results with a recent systematic review on cognitive training, [37] and a meta-analysis comparing multiple interventions on persons with cognitive impairment [38] it is possible that most of the included interventions were either too short or the frequency per week was not high enough to have an effect on global cognition scores. Kallio et al. [37] concluded that interventions with more intense cognitive training had more often beneficial effects on cognition. The association of benefits and total intervention time can also be found in Liang et al. [38], but only for physical exercise interventions.

\section{Non-cognitive symptoms}

There is some evidence that multicomponent interventions positively affect non-cognitive symptoms. All studies that assessed this outcome reported positive effects in this domain. Liang et al. [38] stress that computerized cognitive training was the most effective to reduce non-cognitive symptoms, but they had only one study with that component that actually assessed non-cognitive symptoms. The component that showed the second best effects on non-cognitive symptoms in their meta-analysis was physical exercise. Regarding these results and according to a recent review [4], we assume that physical components are vital for an improvement of non-cognitive symptoms. Several national dementia guidelines (e.g., [39, 40]) recommend non-pharmacological approaches for the treatment of non-cognitive symptoms, if these approaches are available. With these multicomponent interventions practitioners have the opportunity to fulfill the recommendations of the dementia guidelines.

\section{$A D L$}

ADL seem to be an outcome which is not easily influenced by intervention. Only one study found an effect of their intervention [33]. They had an active ADL-training component with a high total intervention time and measured ADL with a performance test. We think these elements of chosen component, total intervention time, and chosen assessments were crucial to identify an effect on ADL. Of the three studies that found no effect, one study with high [28] total intervention time did not utilize an ADL-training component. This finding suggests that specific ADLtraining is important to observe an effect. One study [34] incorporated ADL-training in their intervention, but at medium total intervention time. The fact that this study found no effect on ADL as well, gives us a hint of the importance of total intervention time. There was only one intervention [32] which was comparable to Graessel et al. [33] in the used components and total intervention time. But they assessed the ability to execute ADL through caregivers. As this type of assessment does not resemble real capacity to perform ADL, it is possible that they have missed effects of their intervention. Therefore we suggest that it is important to measure ADL with a performance test if you want to be sensitive to changes. Given these findings, research is needed to investigate our claim on the importance of an ADL-training component and total intervention time.

\section{$Q o L$}

It seems that it is hard to have an impact on Qol with multicomponent interventions, as there was only one study that reported significant effects [28]. We attribute this lack of effects partly to the measures 
used. In two of the studies, the authors used the QoL-AD [28, 31]. One of these studies added the CBS-QoLD [28] and in one, the Short Form 36 health survey questionnaire (SF-36) [34] was solely utilized. The SF-36 is a broad questionnaire on health-related quality of life that asks how the current mental and physical condition, as well as felt pain, limits someone in different activities. The CBS-QoLD strongly resembles a measure of depression. The QoL-AD assesses QoL through asking questions about the living situation or the financial situation. These are aspects that interventions did not aim to change and therefore logically had not affected. Now remains the question, how Maci et al. [28] could have an impact on the QoL-AD and the CBS-QoLD. As their intervention also had a positive effect on depression, it should also improve the CBS-QoLD. The effects on the QOL-AD might be explained as follows: In their intervention, they brought home-dwelling PwD to a gym five times a week for $4 \mathrm{~h}$. There they met new people and were encouraged to socially interact with them. It is safe to assume that they befriended some people of their group. These new friendships could have positively affected items where they are asked to describe their current relationship with friends or their current mood. Additionally, this intervention was a huge respite for the caregivers. Through this respite, it is possible that the relationship between PwD and his caregivers improved what is also being asked in the QoL-AD (relationship to spouse and to family). All of these factors considered, we suggest that $\mathrm{QoL}$ is a construct that is hard to improve because of its broadness and elements that were not part of the interventions. To have an effect on this domain an intervention that drastically changes the current life situation of a PwD toward an active lifestyle with high total intervention time seems to be the most suitable. These interpretations should be regarded very carefully, because the study of Maci et al. [28] was a pilot study with a very small sample size that tested a lot of different outcomes which raises the probability of inflation of the alpha-error. Indeed it is possible that their reported effects could be false positives. Therefore they need to be replicated to strengthen our claims.

\section{Combination of components}

There was a combination of intervention components that most often had effects on different outcome domains. This was the combination of cognitive and physical intervention components. Four out of five studies that included this combination found effects on at least two different outcome domains. Additionally, these studies had moderate to high total intervention time. Furthermore, these effects were found for PwMCI and PwD, partly depending in the dosage/total intervention time. Our findings are supported by a recent meta-analysis that examined the effects of combined cognitive and physical exercise training in persons with cognitive impairment [41]. They found that these combined interventions showed a small-to-medium positive effect on global cognitive function, a moderate-to-large positive effect for ADL and a small-to-medium positive effect for mood. We suggest that these findings can be explained best by the similarity of multicomponent group interventions of moderate to high total intervention time with an active and socially integrated lifestyle. The application of multiple different interventions with a moderate to high total intervention time resembles the active part of the model, while the group setting leads to a social integration of the participants. This lifestyle is regarded as being protective against dementia and therefore is recommended for primary prevention [42]. Hence, it is logical to assume that interventions that resemble this active and socially integrated lifestyle should have a delaying effect on typical dementia decline, even after its onset.

\section{Target group}

Our review might indicate that multicomponent interventions that solely target PwMCI are less effective. There were three studies [27, 29, 30] which only targeted this group and they found no effects on performance tests. We want to emphasize that this finding has to be interpreted with caution, because these studies were largely different in sample size; combination of components; and total intervention time. Consequently further studies are needed to illustrate the effects of multicomponent interventions on PwMCI.

\section{Quality of evidence}

Following Hansen, Jørgensen, \& Ørtenblad's [43] notion that blinding of participants and personnel is impossible in non-pharmacological interventions, we did not evaluate its absence as a dearth of quality. Nevertheless, most of the included studies showed at least one domain with a high risk of bias. Most often, the afflicted domains were reporting and attri- 
tion. This reduces the validity of the results of the included studies.

With 57 participants on average, the included studies have rather small sample sizes for intervention studies in health service research which limits the generalizability of the results onto the group of community-dwelling PwD and PwMCI. Therefore our implications are directed at this group.

\section{Limitations}

There are some limitations that we want to address. We decided to look at the immediate effects of the interventions and therefore excluded follow-up analyses or subgroup analyses from our review. We did this according to drug approval studies and in an attempt to reduce the complexity of this review. However, it is possible and even probable that we missed effects that arise later on in the observation of the participants or affect only some of the participants. Around half of the included studies had an intervention length of three months and less. This period of time might be too short to grasp an effect on some of the collected outcomes. Especially, it might be impossible to create long lasting effects on cognitive impairment with such short intervention lengths. Thus, on the one hand it seems to be important to have longer intervention periods and on the other hand to evaluate the benefit of an intervention on its shortand long-term effects. Additionally, the variations in intervention length increased the heterogeneity of the included studies. We took these into account by calculating a total intervention time score and analyzing its effects.

Another limitation is that there are some pilot studies with a relatively small sample size $[28,29]$. As the $p$-value is dependent on sample size it could be that effects could not be detected or some effects might represent false positives. Therefore high quality studies with big sample sizes are mandatory to strengthen our claims.

To limit the complexity of our review and to reduce heterogeneity between interventions, we excluded studies using occupational therapy, psychotherapeutic elements, and interventions with tailored elements. Due to their special focus on unmet needs of the individual, they are not comparable to interventions which do not individually support the subjects. Most of all, through using individual support, it is impossible to distinguish if the standardized intervention that every subject received, is accountable for the effects or the special individual support. Hence, these interventions (e.g., [44]) should be examined in a separate review. For those reasons, our review is unable to make any statement concerning these special interventions.

We believe that these and other restrictions in our inclusion criteria might be accountable for the different recommendations of our review, compared to the review of Olazaran et al. [9]. They gave multicomponent interventions a grade $\mathrm{B}$ recommendation concerning the same outcomes that we looked at. We suggest that generalized grade B recommendations overreach the effects. Olazaran et al. [9] used a broader definition of multicomponent interventions than us. They even included single-domain interventions with multiple components (e.g., 45]), or interventions solely targeting the caregivers [46] in their review. In our opinion, these studies are too heterogeneous to be subsumed under the same category. Therefore, we argue that Olazaran et al. [9] could have overestimated the effects of multicomponent interventions, because the included studies were too different.

Thus, our review gives a qualitative estimation of the immediate effects of standardized multicomponent group interventions on PwMCI or PwD. In our review we do not intend to give generalized recommendations. We suggest that the complexity of multicomponent interventions requires more detailed recommendations that take five factors into account. First, it is necessary to consider that multicomponent interventions vary in the number of intervention components and their specific combination. We found that the effect of an intervention was greatly dependent on its combined components. Second, it is important to realize that cognitive impairment has various forms from MCI to severe dementia. This factor also influenced the observed effects, so that different recommendations concerning different target groups are needed. Third, the intervention length and the frequency per week, which multiplied with each other, give information about the total intervention time. For some outcomes this total intervention time seems to be crucial. The targeted outcome is the fourth factor that needs to be considered. All three aforementioned factors need to be considered differently, depending on the observed outcome. And fifth it seems to make a difference which assessment you use to assess the outcome. Some assessments might lack the required sensitivity to detect change. Thus, we will give specific recommendations which better reflect the profile of multicomponent interventions. 


\section{Future research}

There is a need for high quality studies examining the additional value of multicomponent interventions over single-component interventions. Such an approach was not used in the included studies. Therefore, we do not exactly know whether the effort of combining interventions is worth it. Furthermore, it should be investigated which components are crucial and should be combined to have the most effect. Hence, studies comparing two singlecomponent interventions and a combination of the two interventions should be conducted, followed by studies doing the same with two-component interventions and three-component interventions and so on. Research on the minimum necessary total intervention time of the multicomponent interventions should then be the next step after identifying crucial components. We found indication that interventions only affect outcomes in people with cognitive impairment above a certain level of dosage. Additionally, studies need to investigate which combinations of intervention components are most suitable for different levels of cognitive impairment.

Furthermore, we think it is crucial to establish a uniform denomination for these interventions which we called multicomponent interventions in this review. In this regard, we propose the term "multi-domain interventions". Through using the word "domain", we want to underline, that these interventions combine different components of different domains with the goal to have an effect on multiple outcomes from different domains (e.g., an intervention that uses cognitive stimulation as well as psychomotor activities). The term multicomponent is used in a broader way and is therefore less precise. For example, it can also mean an intervention with multiple components of only one domain and therefore it can only have an effect on this domain (e.g., an intervention that uses cognitive training as well as cognitive stimulation). On the one hand, using a uniform denomination will have several benefits for researchers. First, researches implementing interventions can be more precise in the kind of intervention they are applying. Second, researches conducting systematic reviews can be more specific in their search terms, resulting in a more accurate detection rate. And third, using a uniform denomination and definition can lead to reviews that reach a consensus on the efficacy of these interventions. On the other hand, it will help people outside of research to more easily access information about these interventions.
Key messages

Our key messages are most relevant for healthcare providers and policy makers. Especially practitioners working at day care centers, who are looking for worthwhile interventions, should consider the following points:

- To positively affect multiple domains, you need to combine at least cognitive and physical interventions with a moderate to high total intervention time. This notion seems to be independent of target group.

- To positively affect non-cognitive symptoms of dementia, a physical intervention component is mandatory. This effect is independent of the target group. No statements can be made concerning total intervention time.

- To positively affect ADL, ADL-training should be part of the intervention. To most sensitively detect change in ADL, it should be assessed with a performance test. No statements can be made concerning the target group.

\section{ACKNOWLEDGMENTS}

We would like to thank Henry Brodaty (H.B.), Mary Mittelman (M.M.), and Richard Schulz (R.S.) for their help with our search terms, Chiara Vetter (C.V.) and Miriam Baron (M.B.) for screening titles and abstracts, Nicolai Cerveny for the acquisition of full texts and Ruben Muniz Schwochert, Javier Olazaran, Francesca Baglio, Verena Buschert, Sandra Bond Chapman, Audette Rackley, and Linda Hynan for transparent and open science conduct through answering questions concerning their interventions and providing data when needed. We thank the Karl and Veronica Carstens Foundation for funding the OptiDem research program and therefore the present article. Additionally we would like to thank all members of the OptiDem research training group for their support, feedback and inspiration during our scientific colloquiums. We thank Nancy Pendergrass for proofreading the manuscript. And we thank Richard Schulz for giving feedback to the project. The present work was performed in partial fulfillment of the requirements for obtaining the degree 'Dr. rer. biol. hum.' by Dominik Özbe.

E.G. and C.D. are authors of an included article [33].

Authors' disclosures available online (https:// www.j-alz.com/manuscript-disclosures/18-0980r1). 


\section{REFERENCES}

[1] McKhann GM, Knopman DS, Chertkow H, Hyman BT, Jack CR, Kawas CH, Klunk WE, Koroshetz WJ, Manly JJ, Mayeux R, Mohs RC, Morris JC, Rossor MN, Scheltens P, Carrillo MC, Thies B, Weintraub S, Phelps $\mathrm{CH}$ (2011) The diagnosis of dementia due to Alzheimer's disease: Recommendations from the National Institute on Aging-Alzheimer's Association workgroups on diagnostic guidelines for Alzheimer's disease. Alzheimers Dement 7, 263-269.

[2] Salomone S, Caraci F, Leggio GM, Fedotova J, Drago F (2012) New pharmacological strategies for treatment of Alzheimer's disease: Focus on disease modifying drugs. $\mathrm{Br}$ J Clin Pharmacol 73, 504-517.

[3] Woods B, Aguirre EP, Spector AE, Orrell M (2012) Cognitive stimulation to improve cognitive functioning in people with dementia (Review). Cochrane Database Syst Rev, CD005562.

[4] Fleiner T, Leucht S, Forstl H, Zijlstra W, Haussermann $P$ (2017) Effects of short-term exercise interventions on behavioral and psychological symptoms in patients with dementia: A systematic review. J Alzheimers Dis 55, 15831594.

[5] Vink AC, Birks JS, Bruinsma MS, Scholten RJ (2004) Music therapy for people with dementia. Cochrane Database Syst Rev, CD003477.

[6] Forrester LT, Maayan N, Orrell M, Spector AE, Buchan LD, Soares-Weiser K (2014) Aromatherapy for dementia. Cochrane Database Syst Rev, CD003150.

[7] Chung JC, Lai CK (2002) Snoezelen for dementia. Cochrane Database Syst Rev, CD003152.

[8] Karkou V, Meekums B (2017) Dance movement therapy for dementia. Cochrane Database Syst Rev 2, CD011022.

[9] Olazarán J, Reisberg B, Clare L, Cruz I, Peña-Casanova J, Del Ser T, Woods B, Beck C, Auer S, Lai C, Spector A, Fazio S, Bond J, Kivipelto M, Brodaty H, Rojo JM, Collins H, Teri L, Mittelman M, Orrell M, Feldmann HH, Muñiz R (2010) Nonpharmacological therapies in Alzheimer's disease: A systematic review of efficacy. Dement Geriatr Cogn Disord 30, 161-178.

[10] Schulz R, Eden J (2016) Families Caring for an Aging America, National Academies of Sciences, Engineering and Medicine, Washington, DC.

[11] Vernooij-Dassen M, Joling K, van Hout H, Mittelman MS (2010) The process of family-centered counseling for caregivers of persons with dementia: Barriers, facilitators and benefits. Int Psychogeriatr 22, 769-777.

[12] Higgins J, Green S (2011) Cochrane Handbook for Systematic Reviews of Interventions Version 5.1.0 [updated March 2011]. The Cochrane Collaboration. Available from http://www.handbook.cochrane.org.

[13] Buettner LL, Ferrario J (1997) Therapeutic recreationnursing team: A therapeutic intervention for nursing home residents with dementia. Annu Ther Recreation 7, 21-93.

[14] Jung Eun Y, Suk Min L, Hee Sung L, Tae Hoon K, Ji Kye $\mathrm{Ng} \mathrm{J}$, Mee Hyang M (2013) The Effects of cognitive activity combined with active extremity exercise on balance, walking activity, memory level and quality of life of an older adult sample with dementia. J Phys Ther Sci 25, 1601-1604.

[15] Lin LC, Yang MH, Kao CC, Wu SC, Tang SH, Lin JG (2009) Using acupressure and montessori-based activities to decrease agitation for residents with dementia: A cross-over trial. J Am Geriatr Soc 57, 1022-1029.
[16] Gates N, Valenzuela M (2010) Cognitive exercise and its role in cognitive function in older adults. Curr Psychiatry Rep 12, 20-27.

[17] Spector A, Orrell M, Woods B (2010) Cognitive Stimulation Therapy (CST): Effects on different areas of cognitive function for people with dementia. Int J Geriatr Psychiatry 25, 1253-1258.

[18] Woods B, Spector AE, Jones CA, Orrell M, Davies SP (2005) Reminiscence therapy for dementia. Cochrane Database Syst Rev, DC001120.

[19] Petermann F, Bahmer J (2009) Psychoedukation. In Lehrbuch der Verhaltenstherapie: Band 3: Störungen im Kindes- und Jugendalter, Schneider S, Margraf J, eds. Springer Berlin Heidelberg, Berlin, Heidelberg, pp. 193-207.

[20] National Institute on Aging, Try These Exercises, https://go4life.nia.nih.gov/exercises, Accessed 08.01.2018.

[21] Pereira C, Rosado H, Cruz-Ferreira A, Marmeleira J (2018) Effects of a 10-week multimodal exercise program on physical and cognitive function of nursing home residents: A psychomotor intervention pilot study. Aging Clin Exp Res 30, 471-479.

[22] World Health Organization (2005) ICF-The International Classification of Functioning, Disability and Health, World Health Organization, Geneva.

[23] Schmidt RF, Willis WD (2007) Encyclopedia of pain, Springer Berlin Heidelberg, Berlin, Heidelberg.

[24] Faulkner KA, Redfern MS, Cauley JA, Landsittel DF, Studenski SA, Rosano C, Simonsick EM, Harris TB, Shorr RI, Ayonayon HN, Newman AB (2007) Multitasking: Association between poorer performance and a history of recurrent falls. J Am Geriatr Soc 55, 570-576.

[25] American Therapeutic Recreation Association (ATRA) (2015) ATRA Definition of Recreational Therapy. 1. Available from https://www.atra-online. com/assets/pdf/SOPDef15.pdf.

[26] Schwenk M, Zieschang T, Oster P, Hauer K (2010) Dual-task performances can be improved in patients with dementia A randomized controlled trial. Neurology 74, 1961-1968.

[27] Suzuki T, Shimada H, Makizako H, Doi T, Yoshida D, Ito K, Shimokata H, Washimi Y, Endo H, Kato T (2013) A randomized controlled trial of multicomponent exercise in older adults with mild cognitive impairment. PLoS One $\mathbf{8}$, e61483.

[28] Maci T, Pira FL, Quattrocchi G, Nuovo SD, Perciavalle V, Zappia M (2012) Physical and cognitive stimulation in Alzheimer Disease. the GAIA Project: A pilot study. Am J Alzheimers Dis Other Demen 27, 107-113.

[29] Rapp S, Brenes G, Marsh AP (2002) Memory enhancement training for older adults with mild cognitive impairment: A preliminary study. Aging Mental Health 6, 5-11.

[30] Troyer AK, Murphy KJ, Anderson ND, Moscovitch M, Craik FI (2008) Changing everyday memory behaviour in amnestic mild cognitive impairment: A randomised controlled trial. Neuropsychol Rehabil 18, 65-88.

[31] Buschert VC, Friese U, Teipel SJ, Schneider P, Merensky W, Rujescu D, Moller HJ, Hampel H, Buerger K (2011) Effects of a newly developed cognitive intervention in amnestic mild cognitive impairment and mild Alzheimer's disease: A pilot study. J Alzheimers Dis 25, 679-694.

[32] Olazarán J, Muñiz R, Reisberg B, Peña-Casanova J, del Ser T, Cruz-Jentoft AJ, Serrano P, Navarro E, García de la Rocha ML, Frank A, Galiano M, Fernández-Bullido Y, Serra JA, González-Salvador MT, Sevilla C (2004) Ben- 
efits of cognitive-motor intervention in MCI and mild to moderate Alzheimer disease. Neurology 63, 2348-2353.

[33] Graessel E, Stemmer R, Eichenseer B, Pickel S, Donath C, Kornhuber J, Luttenberger K (2011) Non-pharmacological, multicomponent group therapy in patients with degenerative dementia: A 12-month randomised, controlled trial. BMC Medicine 9, 129.

[34] Baglio F, Griffanti L, Saibene FL, Ricci C, Alberoni M, Critelli R, Villanelli F, Fioravanti R, Mantovani F, D'amico A, Cabinio M, Preti MG, Nemni R, Farina E (2015) Multistimulation group therapy in Alzheimer's disease promotes changes in brain functioning. Neurorehabil Neural Repair 29, 13-24.

[35] Lundin-Olsson L, Nyberg L, Gustafson Y (1997) "Stops walking when talking" as a predictor of falls in elderly people. Lancet 249, 617.

[36] Clark CM, Sheppard L, Fillenbaum GG, Galasko D, Morris JC, Koss E, Mohs R, Heyman A (1999) Variability in annual Mini-Mental State Examination score in patients with probable Alzheimer disease: A clinical perspective of data from the Consortium to Establish a Registry for Alzheimer's Disease. Arch Neurol 56, 857-862.

[37] Kallio EL, Ohman H, Kautiainen H, Hietanen M, Pitkala K (2017) Cognitive training interventions for patients with Alzheimer's disease: A systematic review. J Alzheimers Dis 56, 1349-1372.

[38] Liang JH, Xu Y, Lin L, Jia RX, Zhang HB, Hang L (2018) Comparison of multiple interventions for older adults with Alzheimer disease or mild cognitive impairment: A PRISMA-compliant network meta-analysis. Medicine (Baltimore) 97, e10744.

[39] National Collaborating Centre for Mental Health (2007) Dementia: A NICE-SCIE Guideline on supporting people with dementia and their carers in health and social care In National Clinical Practice Guideline, National Institute for Health and Clinical Excellence, ed. British Psychological Society, Leicester.
[40] Deuschl G, Maier W (2016) S3-Leitlinie Demenzen. Leitlinien für Diagnostik und Therapie in der Neurologie. Deutsche Gesellschaft für Neurologie.

[41] Karssemeijer EGA, Aaronson JA, Bossers WJ, Smits T, Olde Rikkert MGM, Kessels RPC (2017) Positive effects of combined cognitive and physical exercise training on cognitive function in older adults with mild cognitive impairment or dementia: A meta-analysis. Ageing Res Rev 40, 75-83.

[42] Fratiglioni L, Paillard-Borg S, Winblad B (2004) An active and socially integrated lifestyle in late life might protect against dementia. Lancet Neurol 3, 343-353.

[43] Hansen VN, Jørgensen T, Ørtenblad L (2006) Massage and touch for dementia. Cochrane Database Syst Rev, CD004989.

[44] Vellas B, Carrie I, Gillette-Guyonnet S, Touchon J, Dantoine T, Dartigues JF, Cuffi MN, Bordes S, Gasnier Y, Robert P, Bories L, Rouaud O, Desclaux F, Sudres K, Bonnefoy M, Pesce A, Dufouil C, Lehericy S, Chupin M, Mangin JF, Payoux P, Adel D, Legrand P, Catheline D, Kanony C, Zaim M, Molinier L, Costa N, Delrieu J, Voisin T, Faisant C, Lala F, Nourhashemi F, Rolland Y, Van Kan GA, Dupuy C, Cantet C, Cestac P, Belleville S, Willis S, Cesari M, Weiner MW, Soto ME, Ousset PJ, Andrieu S (2014) MAPT study: A multidomain approach for preventing Alzheimer's disease: Design and baseline data. J Prev Alzheimers Dis 1, 13-22.

[45] Tadaka E, Kanagawa K (2004) A randomized controlled trial of a group care program for commutity-dwelling elderly people with dementia. Japan J Nurs Sci 1, 19-25.

[46] Teri L, Logsdon RG, Peskind E, Raskind M, Weiner MF, Tractenberg RE, Foster NL, Schneider LS, Sano M, Whitehouse P, Tariot P, Mellow AM, Auchus AP, Grundman M, Thomas RG, Schafer K, Thal LJ, Alzheimer's Disease Cooperative Study (2000) Treatment of agitation in AD: A randomized, placebo-controlled clinical trial. Neurology 55, 1271-1278. 